\title{
miR-382 inhibits migration and invasion by targeting ROR1 through regulating EMT in ovarian cancer
}

\author{
HONG TAN $^{1,2}$, QINGNAN HE $^{3}$, GUANHUI GONG ${ }^{2}$, YIXUAN WANG ${ }^{1,2}$, \\ JUANNI LI ${ }^{1,2}$, JUNPU WANG ${ }^{1,2}$, DING ZHU ${ }^{1,2}$ and XIAOYING WU ${ }^{1,2}$ \\ ${ }^{1}$ Department of Pathology, School of Basic Medical Science, Central South University, Changsha, \\ Hunan 410013; ${ }^{2}$ Department of Pathology, Xiangya Hospital, Central South University, \\ Changsha, Hunan $410008 ;{ }^{3}$ Children's Medical Center, The Second Xiangya Hospital, \\ Central South University, Changsha, Hunan 410011, P.R. China
}

Received August 20, 2015; Accepted October 6, 2015

DOI: 10.3892/ijo.2015.3241

\begin{abstract}
Increasing evidence suggests that microRNAs (miRNAs) play a critical role in tumorigenesis. Decreased expression of miR-382 has been observed in various types of cancers. However, the biological function of miRNA-382 in ovarian cancer is still largely unknown. Here, we found miR-382 was downregulated in human ovarian cancer tissues and cell lines. miR-382 inhibited ovarian cancer cell proliferation, migration, invasion and the epithelial-mesenchymal transition (EMT). Furthermore, we identified receptor tyrosine kinase orphan receptor 1 (ROR1) as a target of miR-382, and miR-382 rescued the promotion effect of ROR1 on migration, invasion and EMT process in SKOV3 and COV434 cells. Collectively, these findings revealed that miR-382 inhibits migration and invision by targeting ROR1 through regulating EMT in ovarian cancer, and might serve as a tumor suppressor in ovarian cancer.
\end{abstract}

\section{Introduction}

MicroRNAs (miRNAs) are short non-coding RNA molecules of 19-24 nucleotides (nt) involved in post-transcriptional regulation of genes expression (1). miRNAs participate in many significant biological processes including cell proliferation, apoptosis, migration, invasion, differentiation, initiation and progression of various cancers (2-4). In recent years, miRNAs were investigated in body fluids, such as plasma, serum, urine, and saliva, and in tissues, and used as biomarkers in diverse diseases including many cancers (5). It was reported that miR-382 was downregulated in stage III/ IV epithelial ovarian carcinoma compared with the normal

Correspondence to: Professor Xiaoying Wu, Department of Pathology, School of Basic Medical Science, Central South University, 172 Tongzipo Road, Changsha, Hunan 410013, P.R. China E-mail: xyw2007@csu.edu.cn

Key words: miR-382, ovarian cancer, receptor tyrosine kinase orphan receptor 1, epithelial-mesenchymal transition, migration, invasion group (6,7). Recently, miR-382 was reported downregulated in human ovarian cancer tissues (8). Yet, limited research has been carried out on the function and mechanism of miR-382 in ovarian cancer.

ROR1 belongs to the RTKs, which are a large family of cell surface glycoproteins (9). It was reported that ovarian cancers patients with high expression levels of ROR1 had a higher rate of relapse and a shorter median survival than ovarian cancers patients who expressed low or negligible levels of ROR1, which indicated that ovarian CSCs express ROR1 that contributed to their abilities to form tumors, thus, making ROR1 a potential target for the treatment of ovarian cancer patients (10). Furthermore, others reported the expression of ROR1 was related to malignant characteristics of ovarian cancer, and ROR1 may act as a novel prognostic biomarker in ovarian cancer (11). These studies suggested ROR1 may function crucially in ovarian cancer. However, whether miR-382 targeted ROR1 or not in ovarian cancer cells remained unclear.

We studied the expression, functions and mechanism of miR-382 in ovarian cancer, and the biological functions including cellular proliferation, migration, invasion and epithelial-mesenchymal transition (EMT) process in vitro, as well as the involved molecular mechanisms including its target relationship with ROR1. Our aim was to provide novel insights to improve therapy and prevention of ovarian cancers.

\section{Materials and methods}

Cell culture and transfection. Ovarian cancer cell lines HO8910, A2780, SKOV3, and COV434 were obtained from the American Type Culture Collection (ATCC, Manassas, VA, USA), human ovarian surface epithelium HOSE cells were purchased from Pricells (Wuhan, China) and cultured in DMEM medium containing 10\% fetal bovine serum (FBS). HO8910, A2780, SKOV3 cells were cultured in RPMI-1640 medium containing $10 \%$ FBS and $1 \%$ antibiotic-antimycotic solution (100 U/ml penicillin and $100 \mu \mathrm{g} / \mathrm{ml}$ streptomycin). COV434 cell were cultured in McCoys 5A culture media. Cells were maintained at $37^{\circ} \mathrm{C}$ in a humidified atmosphere containing 
$5 \% \mathrm{CO}_{2}$. Transfection was carried out by Lipofectamine 2000 reagent (Invitrogen). The plasmids of miR-382 mimics (product no. HmiR-AN0480), miR-382 inhibitor (product no. HmiR-AN0480-SN-10), miRNA NC mimics (product no. CmiR-AN0001) and miRNA NC inhibitor (product no. CmiRAN0001-SN) were purchased from Fulen Gen (Guangzhou, China). The plasmid of pCDNA3.1-ROR1 was constructed by AuraGene (Changsha, China).

miRNA target prediction. Prediction of miR-382 target sites was performed by the online software MicroRNA.org - Targets and Expression (http://www.microrna.org/microrna/home.do). The related function of the target was also considered.

Quantitative real-time PCR. Total RNA was extracted by TRIzol (Invitrogen Life Technologies, Carlsbad, CA, USA) methods, and was reverse transcribed as cDNA by using reverse transcription system (Fermentans, Canada). The primers were designed using Primer 5.0, and the forward and reverse primer sequences are showed in Table I. The quantitative real-time PCR (qRT-PCR) was performed using the SYBR Green qPCR (Toyobo, Japan) for studying the quantitative expression of various genes (ROR1, E-cadherin, vimentin, $\mathrm{N}$-cadherin and Snail) according to the manufacturer's protocol. Three repetitions were tested for qRT-PCR. The relative expressions of miR-382 and mRNAs were normalized to those of internal reference $\mathrm{U} 6$ or $\beta$-actin, and were calculated by the $2^{-\Delta \Delta \mathrm{C}}$ method.

MTT assay. Approximately $5 \times 10^{3}$ cells per well were plated into 96-well plates (Costar, USA). After culturing for 24, 48, or $72 \mathrm{~h}$, the cells were treated with fresh serum-free medium and $10 \mu \mathrm{l} /$ well MTT (Biosharp, Hefei, China) solution (10 mg/ $\mathrm{ml}$ in PBS) according to the manufacturer's protocol. DMSO $100 \mu \mathrm{l}$ was added to every well after the incubation for $4 \mathrm{~h}$. Following incubation at $37^{\circ} \mathrm{C}$ for $10 \mathrm{~min}$, the absorbance was measured by microplate reader (Thermo, USA) at $570 \mathrm{~nm}$ at room temperature.

Colony formation assay. Cells were trypsinized and suspended in medium including $0.3 \%$ agar and $10 \%$ serum, then were plated onto a bottom layer with $0.6 \%$ agar. The cells were plated at 300 cells/well into 6-well plates (Costar). The number of colonies were counted after 14 days by Giemsa staining (Solarbio, Beijing, China) via the equation colony forming efficiency $=$ (number of colonies $/$ number of inoculated cells) $\mathrm{x} 100 \%$.

Scratch assay. Cells (closely $1 \times 10^{5}$ cells) were seeded into 12-well plates (Costar), and were incubated at $37^{\circ} \mathrm{C}$ until cells reached a confluence of $\geq 90 \%$. A scratch was generated by use of a sterile $10-\mu l$ pipette tip, and this time was considered as $0 \mathrm{~h}$ for each experiment. Then, at $48 \mathrm{~h}$, photographic images were acquired with an inverted microscope (Motic, Xiamen, China). Thus, migration distance $=$ the gap of $0 \mathrm{~h}$ - the gap of $48 \mathrm{~h}$ the gaps of $0 \mathrm{~h}$, so the migration distances are presented a reverse relationship with the gap of $48 \mathrm{~h}$ (Figs. 3 and 7).

Invasion assay. Invasion assay were performed in 24-well plates with $8-\mu \mathrm{m}$ pore size chamber inserts (BD, Frankin
Table I. Forward and reverse primer sequences of genes.

\begin{tabular}{ll}
\hline Primer & \multicolumn{1}{c}{ Sequences (or product number) } \\
\hline miR-382 & HmiRQP0480 (product number) \\
U6 & HmiRQP9001 (product number) \\
ROR1 & F: AGATCACAGCTGCCTTCACTAT \\
& R: GACATTCTCCAGGATTTCACAT \\
E-cadherin & F: CTCGGCCTGAAGTGACTCGTAAC \\
& R: CAGCAACGTGATTTCTGCATTTC \\
Vimentin & F: GACGCCATCAACACCGAGTT \\
& R: CTTTGTCGTTGGTTAGCTGGT \\
N-cadherin & F: CAGTATCCGGTCCGATCTGC \\
& R: GTCCTGCTCACCACCACTAC \\
Snail & F: GCCTTCAACTGCAAATACTGC \\
& R: CCTCATGTTTGTGCAGGAGA \\
F-actin & F: AGGGGCCGGACTCGTCATACT \\
& R: GGCGGCACCACCATGTACCCT \\
\hline
\end{tabular}

Lakes, NJ, USA). Approximately $1 \times 10^{5}$ cells/well were resuspended in $200 \mu \mathrm{l}$ of medium without fetal bovine serum (FBS; Gibco), and seeded on the upper chamber with the Matrigelcoated membrane. In addition, $500 \mu 1$ medium supplemented with $10 \%$ FBS was added into the lower chamber. After 24-h incubation at $37^{\circ} \mathrm{C}$ and $5 \% \mathrm{CO}_{2}$, the membranes were stained with $0.1 \%$ crystal violet. The cell numbers were counted via an inverted microscope (Motic). Each assay was performed three independent times.

Western blotting. Protein extracts from SKOV3 and COV434 cells were prepared using RIPA lysis buffer (AuraGene) according to the manufacturer's protocol. The protein concentration was confirmed in accordance with the Bradford protein assay reagent (Beyotime Biotechnology, Suzhou, China), and bovine serum albumin was utilized as a standard. Western blot analysis was subsequently carried out to evaluate the levels of ROR1 (1:200, BM0049, ABZOOM), E-cadherin (1:200, YT1453, Immunoway, Changsha, China), vimentin (1:1,000, YT4881, Immunoway), N-cadherin (1:1,000, ab18203, Abcam, China) and Snail (1:200, ab180714, Abcam). Equal amounts of lysate were resolved via SDS-PAGE, and transferred to a PVDF membrane (Millipore, Bedford, MA, USA) through a semidry transfer method. The PVDF membrane was blocked with 5\% non-fat milk in TBST buffer for $2 \mathrm{~h}$ at room temperature, and then was incubated overnight with primary antibodies, and then was incubated for $1 \mathrm{~h}$ using a suitable secondary antibody (1:2,000, Jackson, USA). Electrochemiluminescence was performed with a Gel Documentation and Analysis System.

Luciferase reporter assay. Luciferase assays were performed in SKOV3 and COV434 cells. SKOV3 and COV434 cells were transfected with each of the plasmids [empty vector (MOCK), ROR1 UTR wild-type (WT) and ROR1 UTR mutant (MUT), 
A

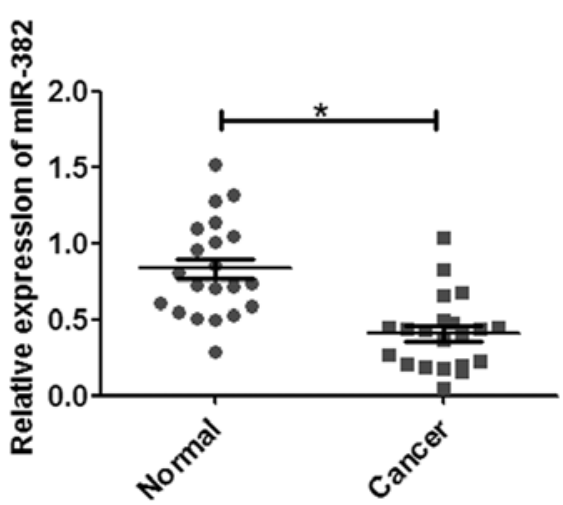

B

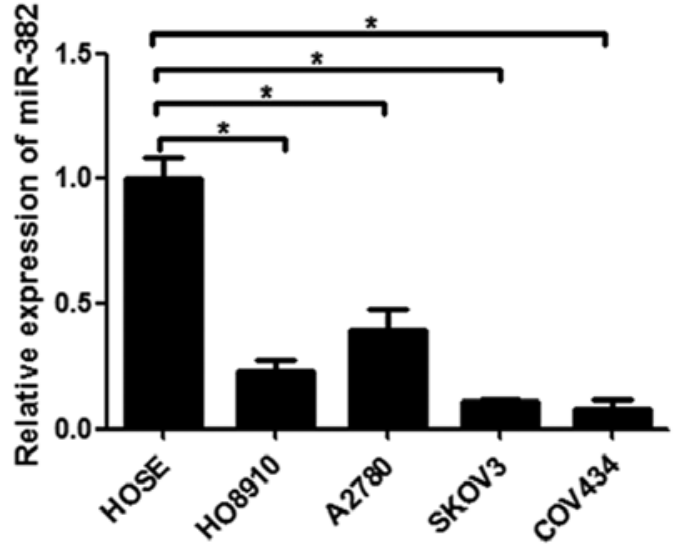

Figure 1. The expression of miR-382 in tissues and cell lines of ovarian cancer. (A) qRT-PCR analysis of miR-382 expression in 20 pairs of ovarian cancer tissues and their corresponding adjacent normal ovarian tissues. The expression of miR-382 was normalized to U6. (B) The relative expression levels of miR-382 were determined by qRT-PCR in human ovarian epithelial cells (HOSE) and ovarian cancer cell lines (HO8910, A2780, SKOV3, COV434). n=20 or 3, " $\mathrm{p}<0.05$.
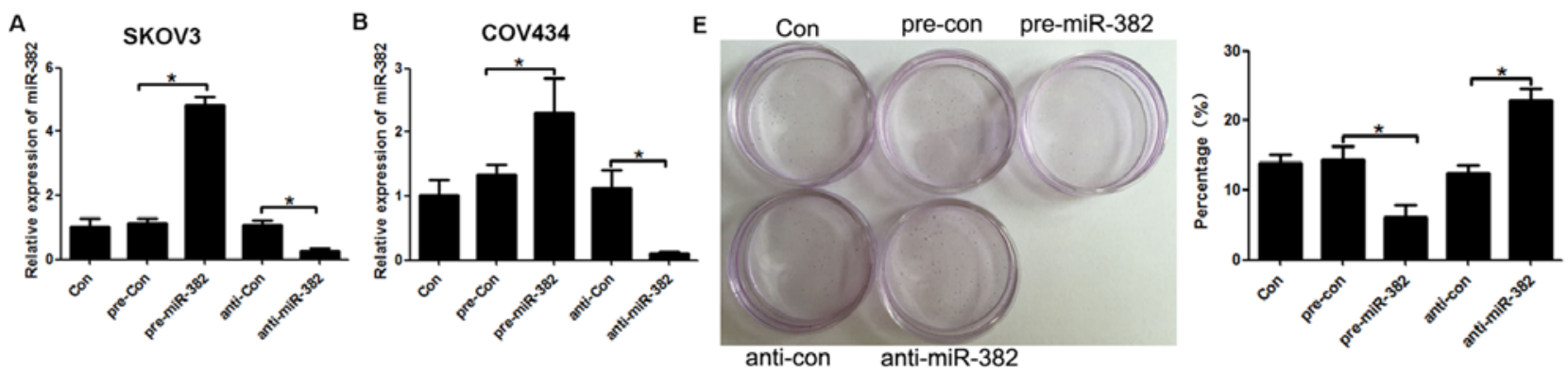

SKov3

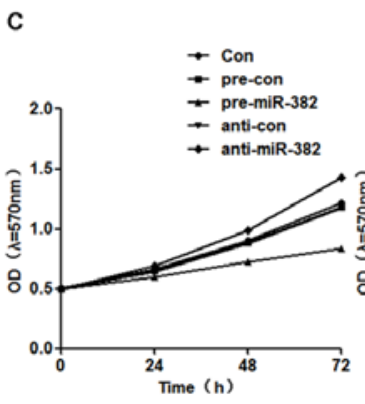

D
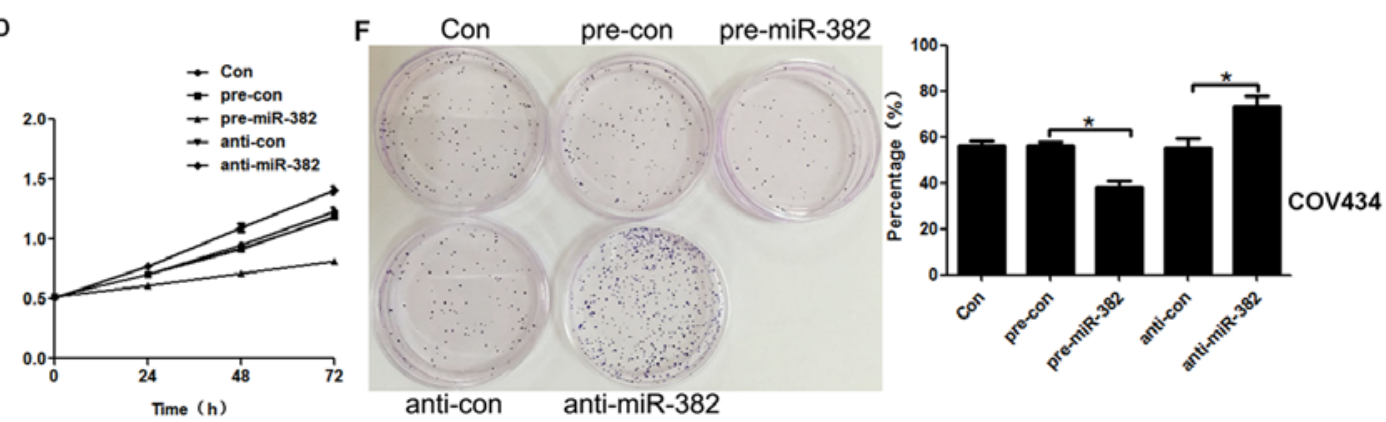

Figure 2. miR-382 inhibits the proliferation of ovarian cancer cells. (A and B) The efficiency of overexpression and interference of miR-382 was validated by qRT-PCR in SKOV3 and COV434 cells. (C and D) miR-382 suppressed the cell proliferation in SKOV3 and COV434 cells by MTT assays. (E and F) miR-382 suppressed cell proliferation in SKOV3 and COV434 cells by colony formation assay for 14 days. $n=3,{ }^{*}<<0.05$.

as a miR-382 binding site] together with miR-382 mimics, miR-382 inhibitor, and negative control RNA in 24-well plates. Two days after transfection, cells were harvested and lysed. Dual luciferase reporter gene assay kit (BioVision, Milpitas, CA, USA) was used to determine the luciferase activities on a luminometer (Roche, Basel, Switzerland). Renilla luciferase activity was normalized to firefly luciferase activity.

Statistical analysis. All statistical tests were conducted with SPSS 17.0 software. The variance between two groups and multiple groups were compared by Student's t-test and ANOVA, respectively. The data are expressed as mean \pm standard deviation (SD). The p-values $<0.05$ were regarded as statistically significant.

\section{Results}

miR-382 is downregulated in human ovarian cancer tissues and cell lines. To investigate the role of miR-382 in ovarian cancer, the expression of miR-382 was examined in tissues and cell lines by qRT-PCR assays. We found the expression of miR-382 was significantly downregulated in human ovarian cancer tissues compared to adjacent non-cancerous tissues $(n=20)$ (Fig. 1A). Moreover, the expression of miR-382 in ovarian cancer cell lines (H08019, A2780, SKOV3 and COV434) was markedly less than in ovarian epithelial cells HOSE (Fig. 1B). These results indicate that miR-382 is downregulated in both human ovarian cancer tissues and cancer cell lines. 
A
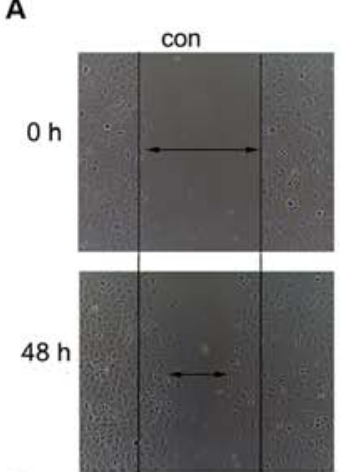

B

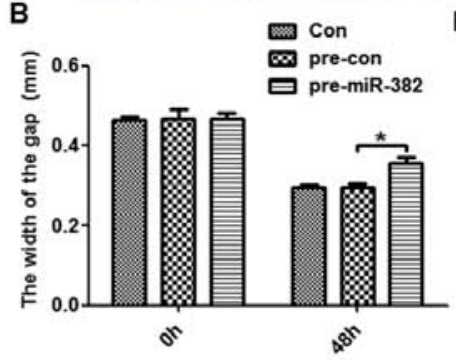

E

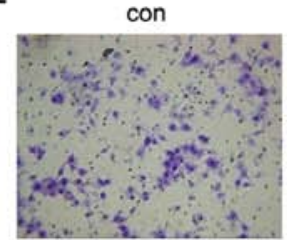

SKOV3
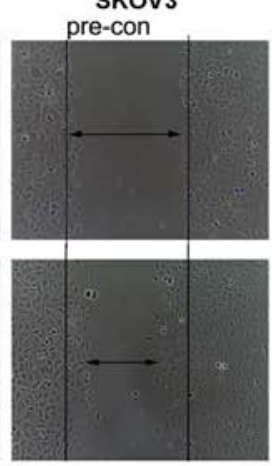

$\mathbf{F}$

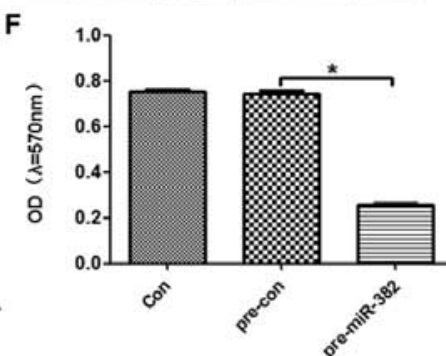

pre-con

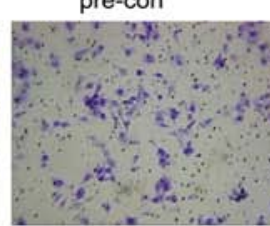

pre-miR-382
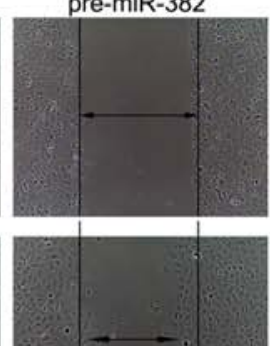

c
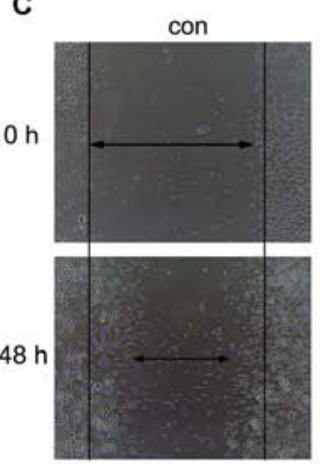

D

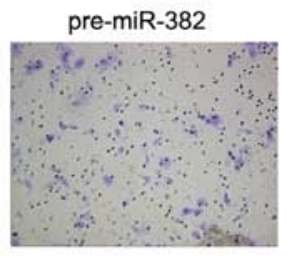

G

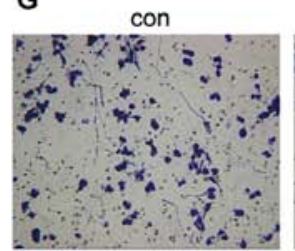

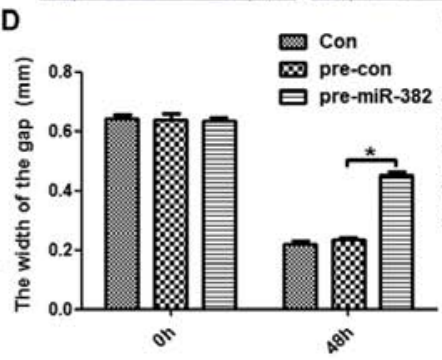
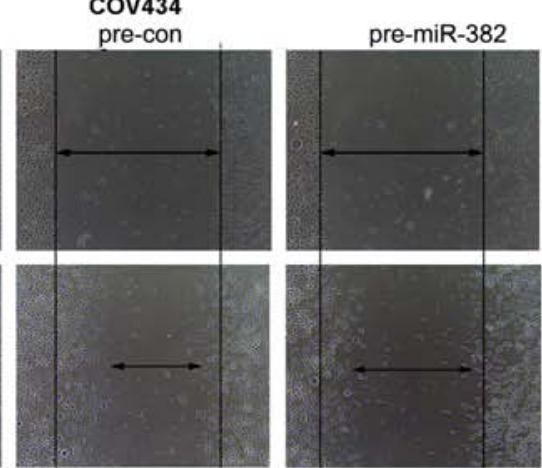

H

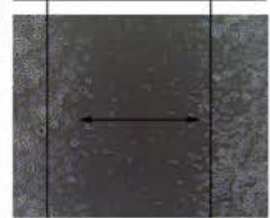

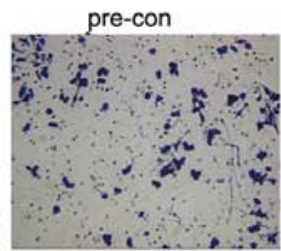

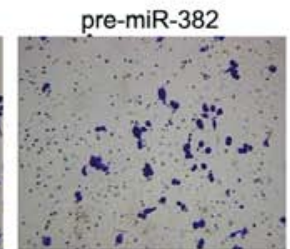

Figure 3. miR-382 inhibits the migration and invasion of ovarian cancer cells. (A and B) miR-382 inhibited the migration in SKOV3 cells as evaluated by wound scratch assay. (C and D) miR-382 suppressed the migration in COV434 cells. (E and F) miR-382 inhibited the invasion in SKOV3 cells in Transwell chamber invasion assays. ( $\mathrm{G}$ and $\mathrm{H}$ ) miR-382 restrained the invasion in COV434 cells in the Transwell assays. $\mathrm{n}=3,{ }^{*} \mathrm{p}<0.05$.

Overexpression of miR-382 suppresses proliferation of ovarian cancer cells in vitro. To better understand the role of miR-382 in ovarian cancer, we used retroviral vectors to establish ovarian cancer cell lines stably overexpressing or silencing miR-382. The expression levels of miR-382 in the SKOV3 and COV434 cell lines were examined by qRT-PCR (Fig. 2A and B). We also measured the growth-promoting effect of miR-382 on ovarian cancer cells by MTT and colony formation assays. MTT assays revealed that overexpression of miR-382 significantly suppressed proliferation of ovarian cancer cells and silencing miR-382 in ovarian cancer cells dramatically promoted proliferation (Fig. 2C and D). In colony formation assay, overexpression of miR-382 significantly inhibited the viability of indicated cells which formed less and smaller clones in SKOV3 (Fig. 2E) and COV434 cells (Fig. 2F). These findings suggest that miR-382 suppresses proliferation of ovarian cancer cells in vitro.

Overexpression of miR-382 suppresses migration and invasion of ovarian cancer cells in vitro. To evaluate the function of miR-382 in the migration and invasion efficiency of ovarian cancer cells, the scratch test and Transwell assays were performed. In scratch assay, overexpression of miR-382 significantly promoted wound healing of SKOV3 (Fig. 3A and B) and COV434 cells (Fig. 3C and D). Moreover, Transwell assay was used to evaluate the invasive ability of ovarian cancer cells. The results indicated that ectopic expres- sion of miR-382 significantly decreased the invasion rate of SKOV3 (Fig. 3E and F) and COV434 cells (Fig. 3G and H).

EMT is taken for a key mechanism by which cancer cells acquire their migratory and invasive capabilities. To further investigate whether the inhibitory effect of miR-382 on migration and invasion was mediated by epithelial to mesenchymal transition (EMT), we examined the expression of several EMT markers by qRT-PCR and western blot assays. Both in mRNA and protein level as expected, miR-382 overexpression increased the expression level of epithelial marker (E-cadherin) and decreased the levels of mesenchymal markers in SKOV3 (Fig. 4A, C and D) and COV434 cells (Fig. 4B, C and E). Taken together, these findings suggest that miR-382 was able to impede invasion mediated by EMT in vitro.

ROR1 is a direct target of miR-382 in ovarian cancer cells. Many miRNAs have been reported to function by binding a specific target. To examine whether miR-382 has a similar mechanism, prediction of miRNA target sites was performed by the online software MicroRNA.org - Targets and Expression (http://www.microrna.org/microrna/home. do), showing the ROR1-3'-UTR regions contain the miR-382 complementary sequence (Fig. 5A). In addition, it has been reported that ROR1 function is crucial in ovarian cancer. Thus, we focused on ROR1 as the primary candidate target of miR-382, and examined firstly the direct binding between 
A

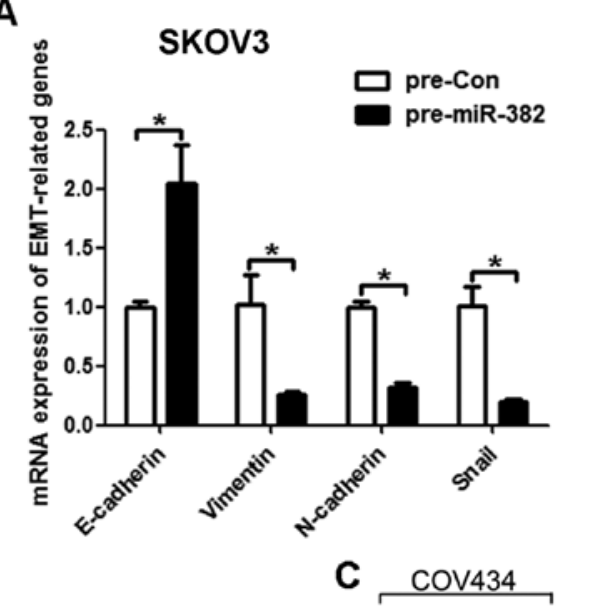

B

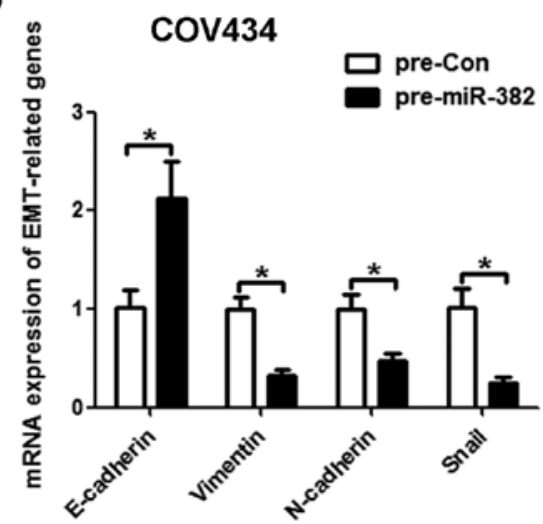

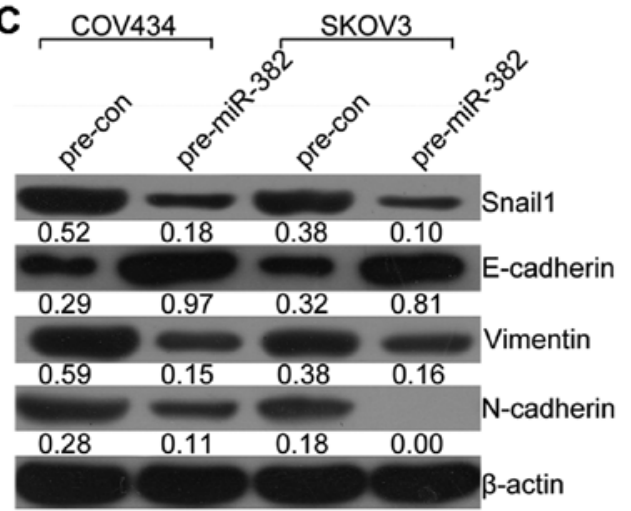
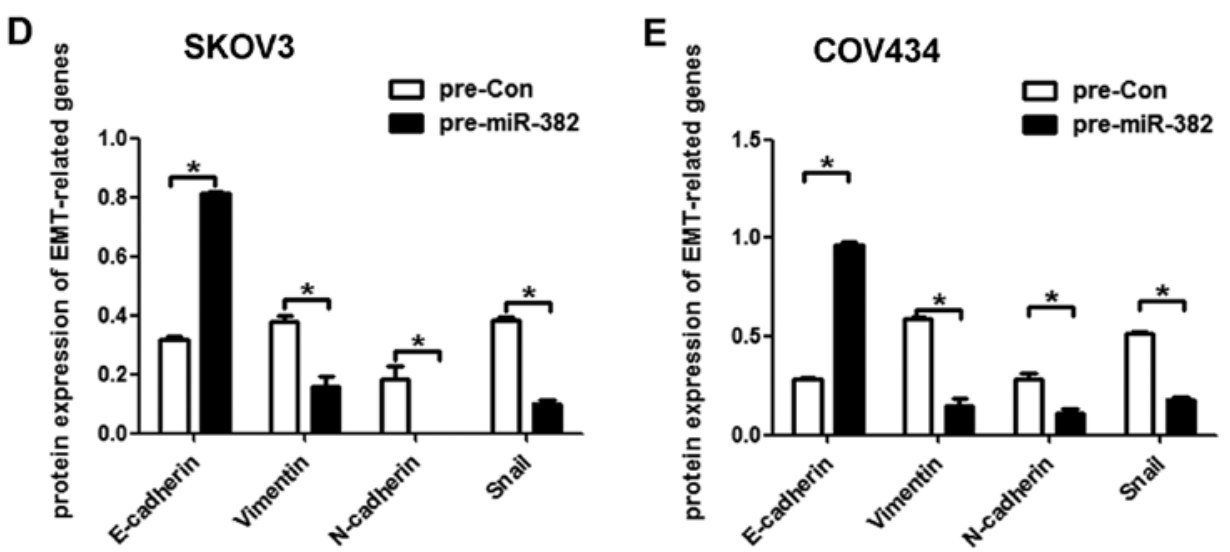

Figure 4. miR-382 suppresses the EMT process in ovarian cancer cells. (A and B) The mRNA expression levels of E-cadherin, vimentin, N-cadherin and Snail when miR-382 was overexpressed in SKOV3 and COV434 cells. (C-E) The protein expression levels of E-cadherin, vimentin, N-cadherin and Snail when miR-382 was overexpressed in SKOV3 and COV434 cells. $n=3,{ }^{*} \mathrm{p}<0.05$.

miR-382 and ROR1, we constructed the vectors ROR13'-UTR and mut-ROR1-3'-UTR to observe their binding activity with miR-382. miR-382 overexpression consistently and significantly reduced the luciferase reporter activity by the ROR1-3'-UTR. However, ROR1-3'-UTR luciferase reporter activity was unaffected by point mutations in the miR-382-binding seed region (Fig. 5B and C). Collectively, these data suggest that miR-382 may inhibit ROR1 expression by targeting its 3'-UTR. As predicted, qRT-PCR and western blot assays showed that, at $48 \mathrm{~h}$ after transfection, the enhanced miR-382 in SKOV3 and COV434 cells significantly repressed ROR1 RNA and protein expression compared to cells transfected with a scrambled control. By comparison, downregulation of miR-382 by inhibitors in SKOV3 (Fig. 5D and F) and COV434 (Fig. 5E and G) cells led to a moderate increase in the ROR1 RNA and protein level. Together, these data provide strong evidence that ROR1 is a specific target of miR-382 in ovarian cancer cells.

miR-382 rescues the promotion effect of ROR 1 on migration and invasion of ovarian cancer cells. To clarify the roles of ROR 1 in ovarian cancer, the expression of ROR1 was investigated in tissues and cell lines. It was found that the mRNA level of ROR1 was upregulated in human ovarian cancer tissues (Fig. 6A), and was negatively correlated with the downregulation of miR-382 (Fig. 6B). The mRNA and protein levels of ROR 1 in the human ovarian cancer cell lines HO8910, SKOV3 and COV434 were markedly higher 
A

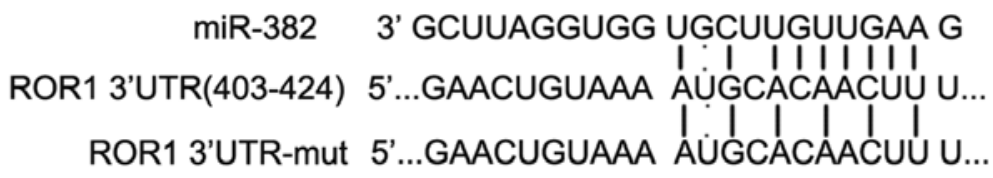
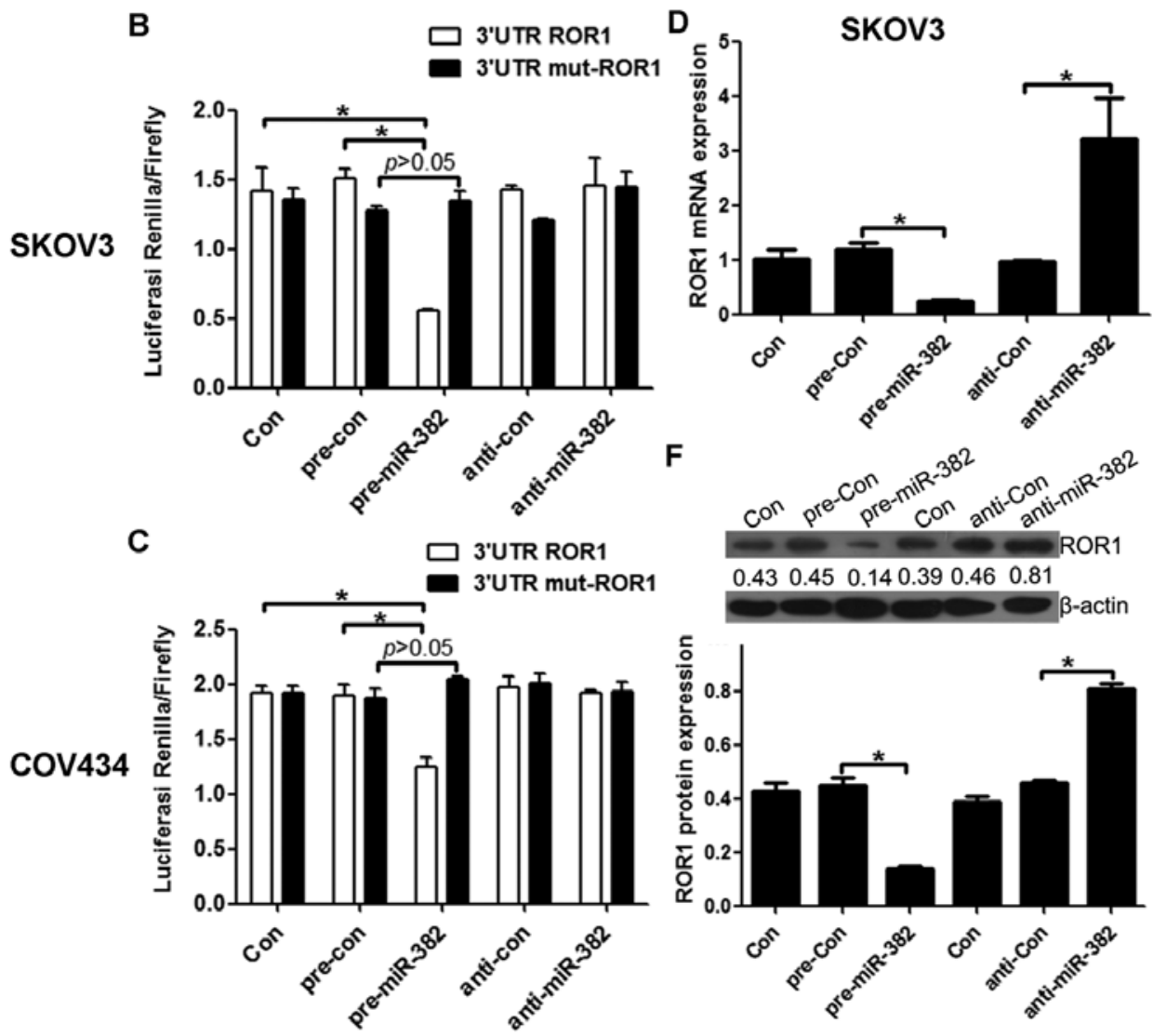
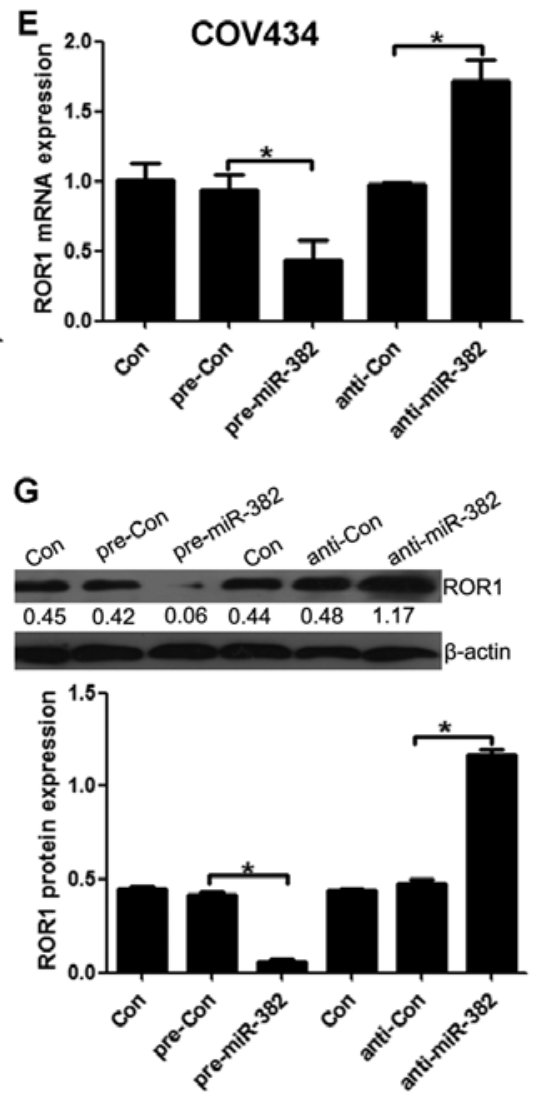

Figure 5. miR-382 targeting ROR1 in ovarian cancer cells. (A) The sequence of the predicted miR-382 binding site and the ROR1-3'-UTR regions containing the wild-type or mutant binding site are displayed. (B and C) Luciferase reporter assay was used to determine whether ROR1 was the direct target of miR-382 in SKOV3 and COV434 cells, respectively. A normal and a mutated 3'-UTR of ROR1 were subcloned into the pRNAT-U6.1/Neo reporter vector. PRNAT-U6.1/ Neo-ROR1-3'-UTR (3'-UTR ROR1-) or PRNAT-U6.1/Neo-mut ROR1-3'-UTR (3'-UTR mut-ROR1) plus pre-miR-382 or anti-miR-382 were cotransfected. The fluorescence intensities were found to mirror the capacity of direct binding. (D and E) The mRNA expression of ROR1 in SKOV3 and COV434 cells with overexpression and interference of miR-382 (pre-miR-382 and anti-miR-382). (F and G) The protein level of ROR1 in SKOV3 and COV434 cells with added pre-miR-382 and anti-miR-382. $\mathrm{n}=3,{ }^{*} \mathrm{p}<0.05$.

than their expression in human ovarian epithelial HOSE cells (Fig. 6C). To evaluate the function of ROR1 that acted as a target of miR-382 in the migration and invasion efficiency of ovarian cancer cells, the scratch test and the Transwel assays were performed. The overexpression of ROR1 significantly increased cell migration, which was rescued by overexpression of miR-382 in both SKOV3 (Fig. 7A and B) and COV434 cells (Fig. 7C and D). Overexpression of ROR1 promoted the cell invasion, while this effect was reversed by overexpression of miR-382 in SKOV3 (Fig. 7E and F) and COV434 cells (Fig. 7G and H). Taken together, these findings suggested that miR-382 inhibited migration and invasion by directly targeting ROR1 in SKOV3 and COV434 cells.

miR-382 is able to rescue the effect of ROR 1 on EMT process of ovarian cancer cells. The above observations suggested that miR-382 could inhibit ovarian cancer cell migration and invasion and exerted its function by directly targeting ROR1; we further investigated the mechanism by which miR-382 and ROR1 regulate the cell malignant phenotype. To determine whether the typical molecular alternations of EMT occurred, the RNA and protein levels of epithelial (E-cadherin), mesenchymal (vimentin and N-cadherin) markers and EMT-related transcription factor Snail were measured by qRT-PCR and western blot assays. ROR1 overexpression reduced the mRNA expression of the epithelial maker E-cadherin and increased the mesenchymal makers $\mathrm{N}$-cadherin, vimentin and EMT-related transcription factor Snail, while the effect was reversed by overexpression of miR-382 in SKOV3 (Fig. 8A) and COV434 cells (Fig. 8B). In line with mRNA expression, ROR1 overexpression lowered the protein amounts of the epithelial maker E-cadherin and increased the expression of the mesenchymal makers $\mathrm{N}$-cadherin, vimentin and EMT-related transcription factor 

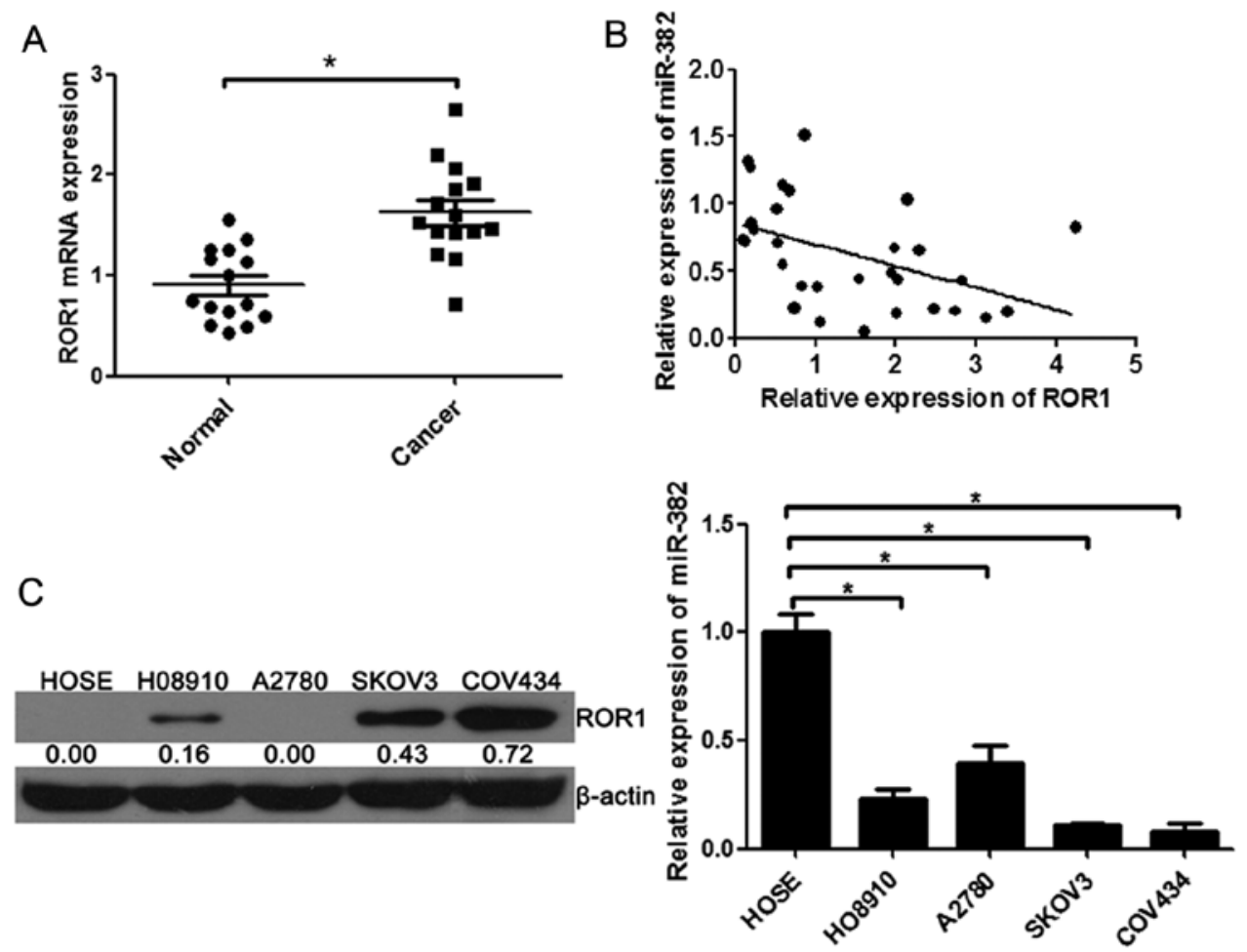

Figure 6. ROR1 is overexpressed and correlated with miR-382 in ovarian cancer tissues and cells. (A) The expression of ROR1 was upregulated in human ovarian cancer tissues. (B) Correlation between expression level of ROR1 and that of miR-382 in 15 matched tissues. Linear regression coefficient and statistical significance is indicated. (C) The mRNA and protein levels of ROR1 were increased in ovarian cancer cell lines. $n=3$ or 15 , ${ }^{*} p<0.05$.

A

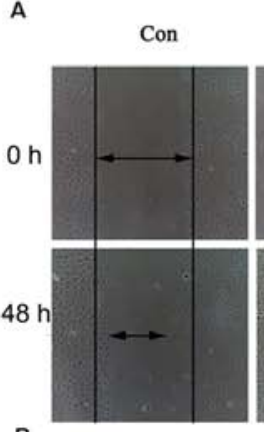

B

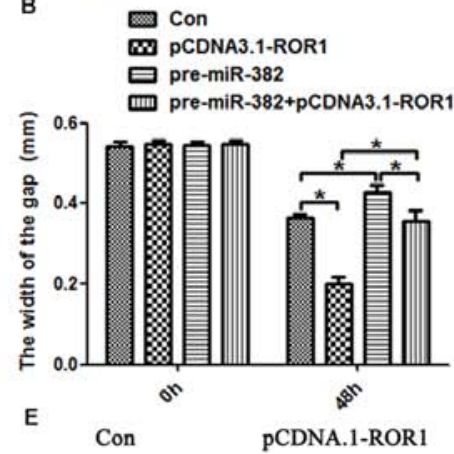

SKov3

pre-miR-382
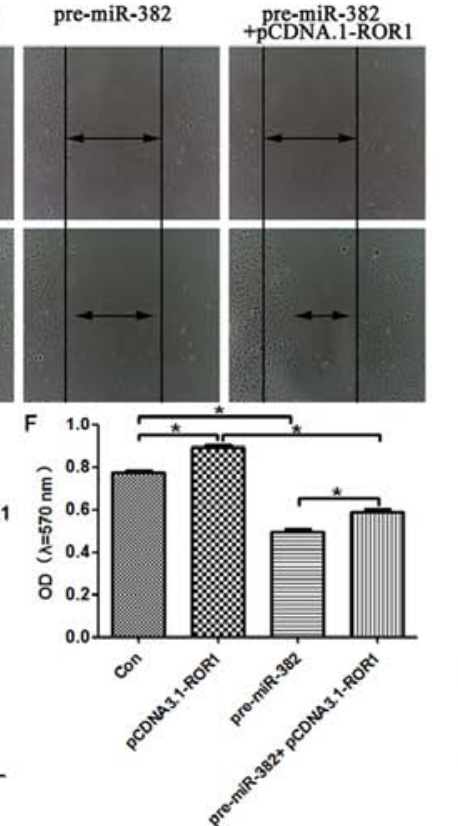

pre-miR-382

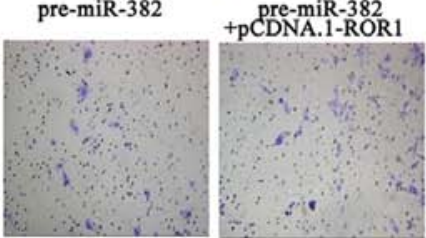

c

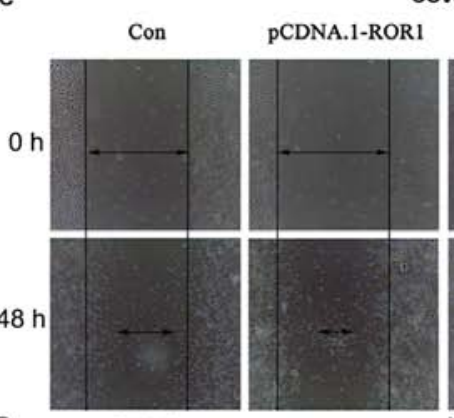

D Con

Con pre-miR-382

E 0.8 血 pre-miR-382+pCDNA3.1-ROR1
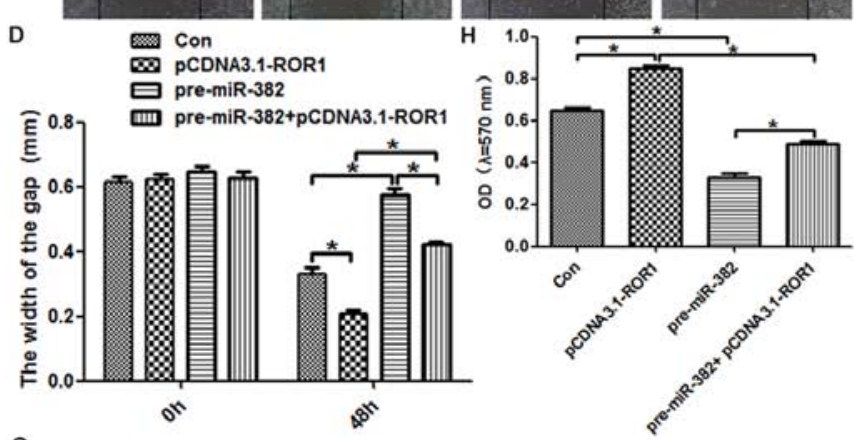

G
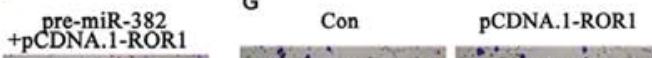
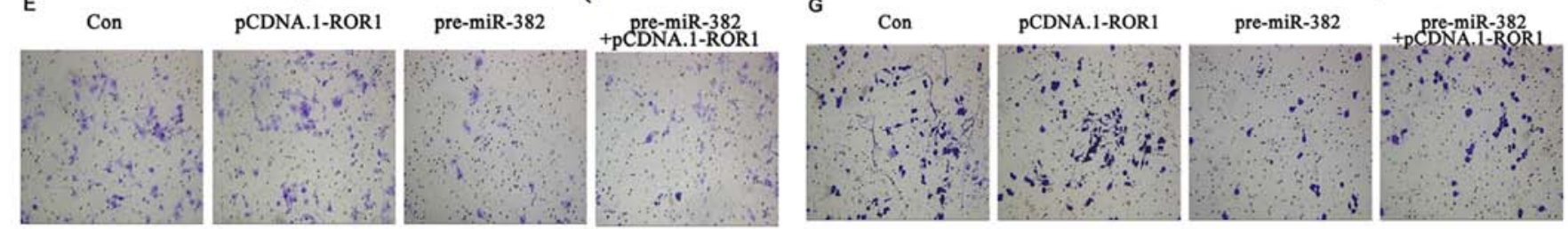

Figure 7. miR-382 rescued the promotion effect of ROR1 on migration and invasion of ovarian cancer cells. (A and B) ROR 1 promoted migration by targeting miR-382 in SKOV3 cells as shown in the wound scratch assay. (C and D) ROR1 promoted migration by targeting miR-382 in COV434 cells in the wound scratch assays, $(\mathrm{E}$ and $\mathrm{F}$ ) and promoted the invasion by targeting to miR-382 in SKOV3 cells. (G and H) miR-382 accelerated the invasion effect of ROR1 by targeting miR-382 in COV434 cells in the Transwell assays. $n=3$, ${ }^{p}<<0.05$. 
A

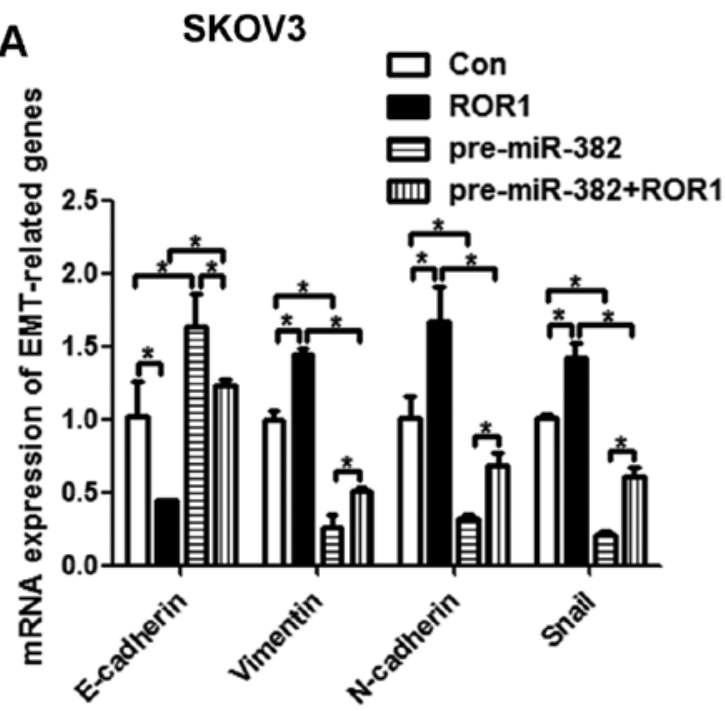

C
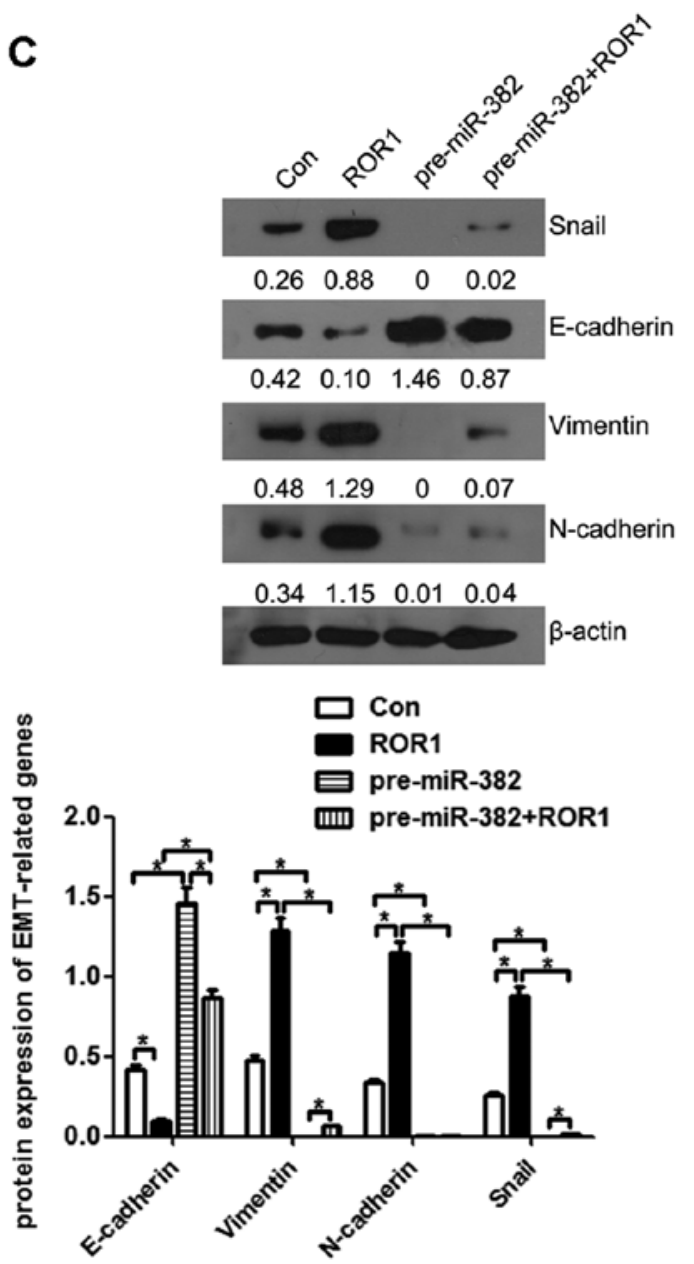

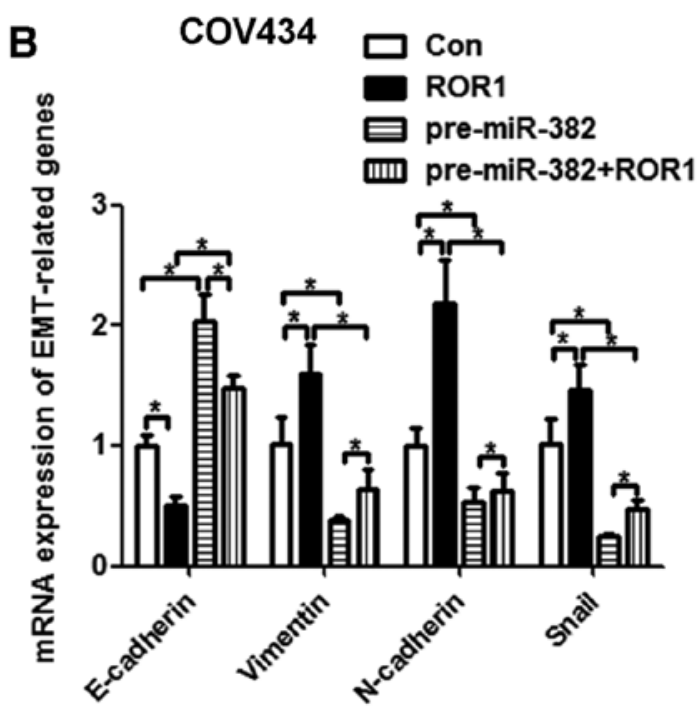

D

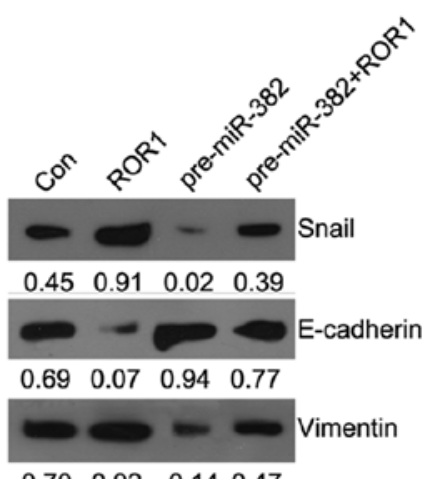

$\begin{array}{llll}0.70 & 0.92 & 0.14 & 0.47\end{array}$

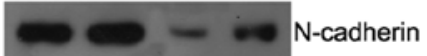

$\begin{array}{llll}0.81 & 0.97 & 0.04 & 0.38\end{array}$

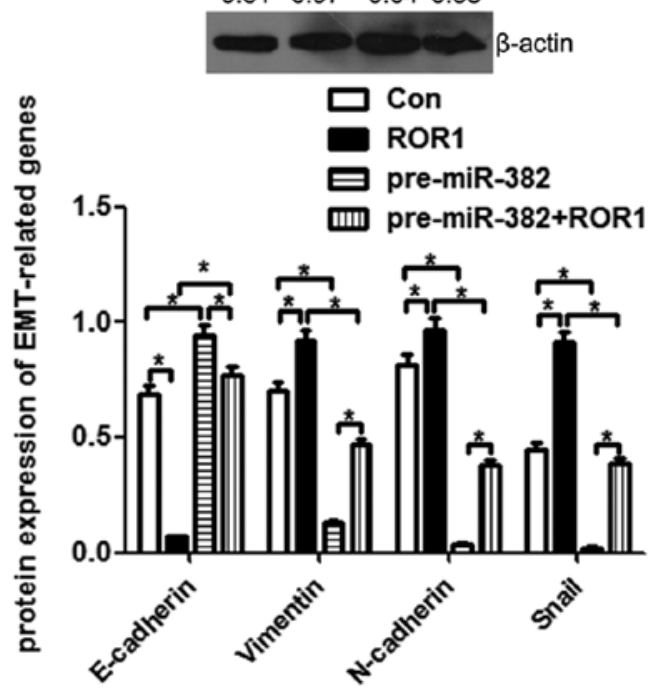

Figure 8. miR-382 affects ovarian cancer cell migration and invasion by ROR1 via regulating the EMT process. (A and B) QRT-PCR assays of the mRNA expression levels of E-cadherin, vimentin, N-cadherin and Snail when miR-382 was overexpressed in SKOV3 and COV434 cells with or without ROR1 restoration. (C and D) Western blot assays of the protein expression levels of E-cadherin, vimentin, N-cadherin and Snail in SKOV3 and COV434 cells when miR-382 was overexpressed with or without ROR1 restoration. $n=3,{ }^{*} \mathrm{p}<0.05$.

Snail, which was reversed markedly by overexpression of miR-382 in SKOV3 (Fig. 8C) and COV434 cells (Fig. 8D). The above findings suggested that miR-382 and its target gene ROR1, could affect ovarian cancer cell migration and invasion by regulating of the EMT process.

\section{Discussion}

Ovarian cancer is the most deadly gynecological malignant tumor, and also the fifth most common cancer death of female in the world $(12,13)$. It is commonly diagnosed at an advanced 
stage, and the overall survival rate of 5 years is $20 \%$ (14). The incidence of ovarian cancer rises along with age, and is most universal at seventy to eighty years of life $(15,16)$. The exact cause of ovarian cancer is still unknown. At present, the conventional therapy for ovarian cancer contains surgery, combining chemotherapy with a platinum-based (such as carboplatin) and a taxane-based (such as paclitaxel) treatment $(17,18)$. Despite the advances in surgery and chemotherapy, the ovarian cancer patients suffer from serious side-effects caused by chemotherapy, and almost $70-80 \%$ of ovarian cancer may recur after first-line chemotherapy (19). Therefore, it is necessary and urgent to find new therapeutic targets that could contribute to the clinical treatment of ovarian cancer.

Increasing evidence exists that miRNAs play an important role in early diagnosis, prognosis, evaluation of therapy results and prevention of cancers (20-23). Each cancer has certain specific miRNA alterations that can be used as a cancer-specific 'signature' for potential clinical application to improve the precision of diagnosis, prognosis and treatment targets $(24,25)$. Depending on the miRNA alterations in cancer of an individual patient, targeted therapies for personalized cancer treatment can be considered (20). Recently, it has been reported that miR-382 was downregulated in human ovarian cancer tissues (8). However, the functions of miR-382 have not previously been explored. Therefore, we studied miR-382 in ovarian cancer. We found that miR-382 was downregulated in human ovarian cancer cells and tissues, which was consistent with a previous study (8). Also, the expression of miR-382 and ROR1 was moderate in ovarian cancer cell lines SKOV3 and COV434, so we selected these cells for our study. Furthermore, we found that miR-382 could inhibit the proliferation, migration, invasion and the EMT process of ovarian cancer cells. These results demonstrated that miR-382 may act as a tumor suppressor ovarian cancer.

Many miRNAs have been reported to function by binding a specific target. We predicted that the miR-382 has potential to bind ROR 1 by bioinformatics methods. Given this, we selected ROR1 as a candidate target of miR-382, and found that miR-382 could target ROR1 by luciferase reporter assay. Overexpression of miR-382 treatment declined the expression of ROR1 and silencing of miR-382 raised the expression of ROR1 in SKOV3 and COV434 cells. These results suggested that ROR 1 could act as a target of miR-382. Furthermore, we showed miR-382 can rescue the acceleration effect of ROR 1 on the proliferation, migration and invasion in SKOV3 and COV434 cells. These results indicated that miR-382 suppressed cancer progression by targeting ROR1 in ovarian cancer.

EMT is a vital step in the invasion and metastasis of cancers, in which epithelial cells lose their apical-basal polarity and cell-cell adhesion as well as gain migratory and invasive properties $(26,27)$. A recent study has shown that the overexpression of miR-382 suppressed the EMT process, while inhibition of miR-382 stimulated EMT in osteosarcoma (28). Cui et al (29) reported breast adenocarcinomas expressing high levels of ROR1 were more likely to have gene expression signatures associated with the EMT and higher rates of metastasis than breast adenocarcinomas expressing low levels of ROR1, suggesting ROR1 may regulate EMT and metastasis. We speculated that both miR-382 and ROR1 were involved in the EMT process. Thus, we determined whether ROR1 that acted as a target of miR-382 was involved in the EMT process in ovarian cancer cells, and found that miR-382 rescued the promotion effect of ROR1 on EMT in SKOV3 and COV434 cells.

In conclusion, miR-382 inhibited the migration and invasion of ovarian cancer cells through targeting ROR1 via regulating EMT process in ovarian cancer cells. miR-382 functioned as a tumor suppressor, and might be useful in therapeutics of ovarian cancer.

\section{Acknowledgements}

This study was supported by grants from the National Natural Science Foundation of China (no. 81172469), Science and Technology Program of Hunan Provincial Science Technology Department (no. 2014FJ3090) and Science and Technology Program of Changsha City Science Technology Bureau (no. K1403050-31).

\section{References}

1. Macha MA, Seshacharyulu P, Krishn SR, Pai P, Rachagani S Jain $\mathrm{M}$ and Batra SK: MicroRNAs (miRNAs) as biomarker(s) for prognosis and diagnosis of gastrointestinal (GI) cancers. Curr Pharm Des 20: 5287-5297, 2014.

2. Ebert MS and Sharp PA: Roles for microRNAs in conferring robustness to biological processes. Cell 149: 515-524, 2012.

3. Ruepp A, Kowarsch A and Theis F: PhenomiR: microRNAs in human diseases and biological processes. Methods Mol Biol 822: 249-260, 2012.

4. Tüfekci KU, Meuwissen RL and Genç S: The role of microRNAs in biological processes. Methods Mol Biol 1107: 15-31, 2014.

5. Tricoli JV and Jacobson JW: MicroRNA: Potential for cancer detection, diagnosis, and prognosis. Cancer Res 67: 4553-4555, 2007.

6. Dahiya N and Morin PJ: MicroRNAs in ovarian carcinomas. Endocr Relat Cancer 17: F77-F89, 2010.

7. Wyman SK, Parkin RK, Mitchell PS, Fritz BR, O'Briant K, Godwin AK, Urban N, Drescher CW, Knudsen BS and Tewari M: Repertoire of microRNAs in epithelial ovarian cancer as determined by next generation sequencing of small RNA cDNA libraries. PLoS One 4: e5311, 2009.

8. Thériault BL, Basavarajappa HD, Lim H, Pajovic S, Gallie BL and Corson TW: Transcriptional and epigenetic regulation of KIF14 overexpression in ovarian cancer. PLoS One 9: e91540, 2014.

9. Gschwind A, Fischer OM and Ullrich A: The discovery of receptor tyrosine kinases: Targets for cancer therapy. Nat Rev Cancer 4: 361-370, 2004.

10. Zhang S, Cui B, Lai H, Liu G, Ghia EM, Widhopf GF II, Zhang Z, Wu CC, Chen L, Wu R, et al: Ovarian cancer stem cells express ROR1, which can be targeted for anti-cancer-stem-cell therapy. Proc Natl Acad Sci USA 111: 17266-17271, 2014.

11. Zhang H, Qiu J, Ye C, Yang D, Gao L, Su Y, Tang X, Xu N, Zhang D, Xiong L, et al: ROR1 expression correlated with poor clinical outcome in human ovarian cancer. Sci Rep 4: 5811, 2014.

12. Böcker W: WHO classification of breast tumors and tumors of the female genital organs: Pathology and genetics. Verh Dtsch Ges Pathol 86: 116-119, 2002 (In German).

13. Kan CW, Howell VM, Hahn MA and Marsh DJ: Genomic alterations as mediators of miRNA dysregulation in ovarian cancer. Genes Chromosomes Cancer 54: 1-19, 2015.

14. Vargas-Hernández VM, Moreno-Eutimio MA, AcostaAltamirano $\mathrm{G}$ and Vargas-Aguilar VM: Management of recurrent epithelial ovarian cancer. Gland Surg 3: 198-202, 2014.

15. Goff BA, Mandel L, Muntz HG and Melancon CH: Ovarian carcinoma diagnosis. Cancer 89: 2068-2075, 2000.

16. Zhou XM, Zhang $\mathrm{H}$ and Han X: Role of epithelial to mesenchymal transition proteins in gynecological cancers: Pathological and therapeutic perspectives. Tumour Biol 35: 9523-9530, 2014.

17. Wang DH, Guo L and Wu XH: Checkpoint inhibitors in immunotherapy of ovarian cancer. Tumour Biol 36: 33-39, 2015.

18. Muccioli M and Benencia F: Toll-like Receptors in ovarian cancer as targets for immunotherapies. Front Immunol 5: 341, 2014.

19. Garces AH, Dias MS, Paulino E, Ferreira CG and de Melo AC: Treatment of ovarian cancer beyond chemotherapy: Are we hitting the target? Cancer Chemother Pharmacol 75: 221-234, 2015. 
20. Sethi S, Ali S, Sethi S and Sarkar FH: MicroRNAs in personalized cancer therapy. Clin Genet 86: 68-73, 2014.

21. Casanova-Salas I, Rubio-Briones J, Calatrava A, Mancarella C, Masiá E, Casanova J, Fernández-Serra A, Rubio L, RamírezBackhaus M, Armiñán A, et al: Identification of miR-187 and miR-182 as biomarkers of early diagnosis and prognosis in patients with prostate cancer treated with radical prostatectomy. J Urol 192: 252-259, 2014.

22. Jiang C, Chen X, Alattar M, Wei J and Liu H: MicroRNAs in tumorigenesis, metastasis, diagnosis and prognosis of gastric cancer. Cancer Gene Ther 22: 291-301, 2015.

23. Li SQ, Chen FJ and Cao XF: Distinctive microRNAs in esophageal tumor: Early diagnosis, prognosis judgment, and tumor treatment. Dis Esophagus 26: 288-298, 2013.

24. Gounaris-Shannon $S$ and Chevassut T: The role of miRNA in haematological malignancy. Bone Marrow Res 2013: 269107, 2013.

25. Sethi S, Kong D, Land S, Dyson G, Sakr WA and Sarkar FH: Comprehensive molecular oncogenomic profiling and miRNA analysis of prostate cancer. Am J Transl Res 5: 200-211, 2013.
26. Koutsaki M, Spandidos DA and Zaravinos A: Epithelialmesenchymal transition-associated miRNAs in ovarian carcinoma, with highlight on the miR-200 family: Prognostic value and prospective role in ovarian cancer therapeutics. Cancer Lett 351: 173-181, 2014.

27. Takai M, Terai Y, Kawaguchi H, Ashihara K, Fujiwara S, Tanaka T, Tsunetoh S, Tanaka Y, Sasaki H, Kanemura M, et al: The EMT (epithelial-mesenchymal-transition)-related protein expression indicates the metastatic status and prognosis in patients with ovarian cancer. J Ovarian Res 7: 76, 2014.

28. Xu M, Jin H, Xu CX, Sun B, Song ZG, Bi WZ and Wang Y: miR-382 inhibits osteosarcoma metastasis and relapse by targeting Y box-binding protein 1. Mol Ther 23: 89-98, 2015.

29. Cui B, Zhang S, Chen L, Yu J, Widhopf GF II, Fecteau JF, Rassenti LZ and Kipps TJ: Targeting ROR1 inhibits epithelialmesenchymal transition and metastasis. Cancer Res 73: 3649-3660, 2013. 